\title{
FEM-PGD BASED TECHNIQUE FOR COLUMN SHAPE OPTIMIZATION AGAINST BUCKLING
}

\author{
Tosporn Prasertsri ${ }^{*}$, Supin Sartbumrung ${ }^{2}$, Suneenut Suewongprayoon $^{2}$, \\ Natdanai Sinsamutpadung ${ }^{3}$, and Jaroon Rungamornrat ${ }^{2}$ \\ ${ }^{1}$ Department of Civil Engineering, Faculty of Engineering and Architecture, \\ Rajamangala University of Technology Tawan-ok, Bangkok, Thailand, e-mail: tosporn_pr@ @rmutto.ac.th \\ ${ }^{2}$ Department of Civil Engineering, Faculty of Engineering, Chulalongkorn University, Bangkok, Thailand \\ ${ }^{3}$ Department of Civil Engineering, School of Engineering, \\ King Mongkut's Institute of Technology Ladkrabang, Bangkok, Thailand
}

Received Date: January 25, 2021; Revised Date: May 15, 2021; Acceptance Date: September 8, 2021

\begin{abstract}
This paper presents a simple numerical procedure based upon the projected gradient descent (PGD) and finite element method (FEM) for the shape optimization of laterally restrained columns to attain the maximum elastic buckling load under the specified volumetric constraint. The analysis of the buckling load is achieved via the formulation based on Euler-Bernoulli beam theory, the discretization by the standard finite element technique, and the determination of the least eigenvalue and the corresponding eigenvector via the power method with Rayleigh quotient. In the optimization, the profile of the crosssectional area of the column is represented by piecewise polynomial interpolation functions. The gradient information and the projection operator required in PGD iterations are obtained explicitly in a closed form. A selected set of results is reported to demonstrate not only the good convergence behavior and accuracy of numerical solutions, but also the capability of the proposed technique to attain the optimal shape of columns for various scenarios.
\end{abstract}

Keywords: Elastic buckling, Finite element method, Power method, Projected gradient descent, Shape optimization

\section{Introduction}

Structural optimizations have been a subject of interest and attracted various investigators for many decades [1]. Optimal solutions with the primary objective to enhance the structural performance with the lowest cost are found increasingly important in the design procedure due to the rapid growth of the analysis and design approaches, material sciences (e.g., functionally graded (FG) and advanced materials), and fabrication/construction techniques (e.g., 3D printings). Such advanced technologies start fading away the limitation on the structural configurations and shapes or even the material distribution in the practical design.

A vast number of studies on structural optimization has been recognized in the literature including those related to the development and implementation of techniques to estimate the optimal solutions for various types of structures including trusses [2-8], frames [914], and bridges [15-18]. Among those existing research works, structural shape optimization, which aims mainly to find the shape of structural components that offers the best specified performance under the given constraints, is also one gaining significant attention. An important example and also the main focus of the present study is to find the shape of the strongest column against the buckling capacity. The analytical solution for the optimal shape of the pinned-pinned 
column to attain the maximum elastic buckling load was derived in [19]. A series of analytical solutions for columns with various end conditions (e.g., clamped-free, clamped-clamped, and clamped-hinged columns) and the single modal formulation were also established in the subsequent study [20]. Due to the limitation of analytical techniques in handling large-scale and complex structures, various numerical methods were also proposed for determining the shape of the strongest column. A finite element method together with the iterative process to meet the optimality condition was proposed in [21] to determine the optimal shape of the strongest column subjected to mixed boundary conditions and the minimum area requirement. The single and bimodal optimum buckling loads of the clamped-clamped column was fully investigated via the technique of numerical integration and reiterating procedure in [22] and the need of bimodal formulation was concluded when the area constraint is less than the threshold value. Later, both analytical and numerical techniques were utilized by [23] along with the single and bimodal formulations to further reinvestigate the characteristic of the optimal buckling load of the clamped-clamped column. A technique based on the finite element method and the iterative procedure with the incremental update of the cross-sectional area via the sensitivity number was introduced by [24] to determine the optimal design of columns and frames to attain the maximum buckling capacity. Both bimodal and multimodal features were also treated in their study. A semi-analytical technique based on the representation of columns as a discrete linkspring system (Hencky bar-chain model (HBM)) and the recursive iteration was proposed by [25] to obtain the maximum buckling load of clamped-spring and pinned-spring columns. The technique has been further improved by [26] and extended its applications to the clamped-free column under the uniformly distributed load. In addition, the analytical solutions for the uniform HBM under the axial load and uniform weight and the nonuniform HBM under the axial load were also derived. Evolutionary algorithms such as genetic algorithm were also implemented together with HBM to investigate the maximum buckling load of columns [27]. While ones can enjoy the merit of the HBM-based techniques in which there is no need to directly handle governing differential equations for buckling, the simple discretization of the continuous deformable member into rigid links and the treatment of member flexibility through the rotational springs still requires a significant number of segments to accurate capture the nonuniform variation of the cross-sectional area at the optimal condition.

The present study aims mainly to propose an alternative, simple algorithm for determining the optimal shape of columns with interior lateral restraints to attain the maximum fundamental buckling load. To balance among the simplicity, the accuracy of solutions, and the computational effort, a conventional finite element method (FEM) is selected to discretize the governing equation for the buckling load, a power method with Rayleigh quotient is adopted to compute the least eigenvalue and its corresponding eigenvector of the discretized system, and the projected gradient descent (PGD) scheme is implemented to iterate for the optimal solution under the specified volumetric constraint. To prove the concept, the technique is implemented and demonstrating examples are chosen within the context of a single modal formulation. All essential components established in the present study should be readily extended to handling cases with either bimodal or multimodal formulations.

\section{Problem Formulation}

Consider a perfectly straight column of length $l$ and subjected to the compression force $P$ as shown schematically in Figure 1. The column is subjected to various end conditions such as the clamped (C) end with the full restraint on the transverse displacement and rotation, the hinged 
$(\mathrm{H})$ end with the fully restraint only on the transverse displacement, and the free $(\mathrm{F})$ end without any restraint on the transverse displacement and rotation. The column can be internally restrained against the transverse displacement by a distributed, translational elastic spring with the spring constant $k(x)$ where $x$ is a selected coordinate along the axis of the column with $x=0$ representing the bottom end. The column is made of a homogeneous, isotropic, linearly elastic material with Young's modulus $E$ and possesses a solid circular cross section with the area $A=A(x)$. Let $P \equiv P_{c r}$ denote the elastic flexural buckling load of this particular column (i.e., the least compression force $P$ at the bifurcation equilibrium states). The problem statement, here, is to determine the distribution of the cross-sectional area $A=A(x)$ that maximize the buckling load $P_{c r}$ of the column subjected to the following volumetric constraint:

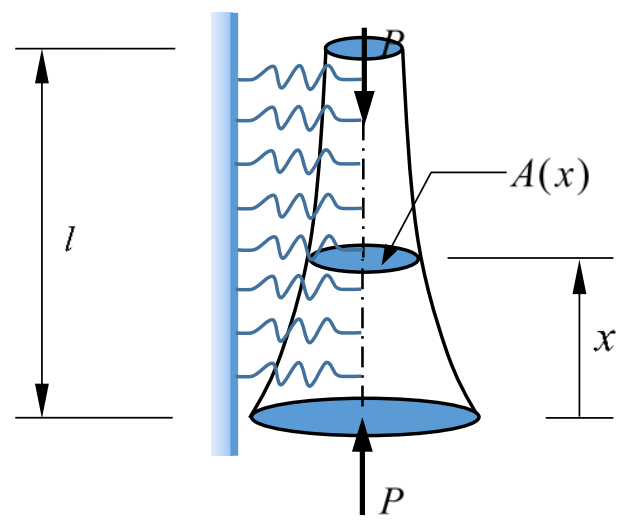

Figure 1. Schematic of a perfectly straight column considered in the present study

$$
\int_{0}^{l} A(x) d x=V_{0}
$$

where $V_{0}$ is a given volume of the constituting material.

The response of the column at the onset of the flexural buckling is modeled by EulerBernoulli beam theory together with assumption on the inextensibility of the axis of the column. In particular, the shear force $V$, the bending moment $M$, the curvature $\kappa$, the rotation $\theta$, and the transverse displacement (or deflection) $v$ at any cross section of the column (located at the coordinate $x$ ) are governed by:

$$
\begin{gathered}
\frac{d V}{d x}+k v=0 ; \quad \frac{d M}{d x}+P \frac{d v}{d x}=V \\
M=E I \kappa=\frac{E A^{2}}{4 \pi} \kappa \\
\kappa=\frac{d \theta}{d x} ; \quad \theta=\frac{d v}{d x}
\end{gathered}
$$


where $I=A^{2} / 4 \pi$ denotes the moment of inertia of the circular cross section. Combining Equation (2)-(4) leads to a homogeneous, linear, fourth-order, ordinary differential equation governing the deflection $v=v(x)$ at the onset of the buckling:

$$
\frac{d^{2}}{d \bar{x}^{2}}\left(\bar{A}^{2} \frac{d^{2} \bar{v}}{d \bar{x}^{2}}\right)+\bar{P} \frac{d^{2} \bar{v}}{d \bar{x}^{2}}+\bar{k} \bar{v}=0
$$

where $\bar{x}=x / l, \bar{v}=v / l, \bar{A}=A l / V_{0}, \bar{P}=4 \pi P l^{4} / E V_{0}^{2}$, and $\bar{k}=4 \pi k l^{6} / E V_{0}^{2}$. In addition to satisfying the governing Equation (5), the deflection $v=v(x)$ must also satisfy the boundary conditions at both ends as listed below for different types of end restraints:

$$
\begin{aligned}
& \text { C-End: } \quad \bar{v}=0, \frac{d \bar{v}}{d \bar{x}}=0 \\
& \text { H-End: } \quad \bar{v}=0, \frac{d^{2} \bar{v}}{d \bar{x}^{2}}=0
\end{aligned}
$$

$$
\text { F-End: } \quad \frac{d^{2} \bar{v}}{d \bar{x}^{2}}=0, \frac{d}{d \bar{x}}\left(\bar{A}^{2} \frac{d^{2} \bar{v}}{d \bar{x}^{2}}\right)+\bar{P} \frac{d \bar{v}}{d \bar{x}}=0
$$

The weak form of the governing differential Equation (5) established via the standard weighted residual technique, the integration-by-parts procedure, and the proper enforcement of the boundary conditions is given by:

$$
\int_{0}^{1} \bar{A}^{2} \frac{d^{2} \bar{w}}{d \bar{x}^{2}} \frac{d^{2} \bar{v}}{d \bar{x}^{2}} d \bar{x}+\int_{0}^{1} \bar{k} \bar{w} \bar{v} d \bar{x}-\bar{P} \int_{0}^{1} \frac{d \bar{w}}{d \bar{x}} \frac{d \bar{v}}{d \bar{x}} d \bar{x}=0
$$

where $\bar{w}$ is any weight function satisfying the integrability condition and the homogeneous essential boundary conditions. For a given profile of the normalized cross-sectional area $\bar{A}=\bar{A}(x)$, the normalized buckling load of the column is, therefore, the least normalized compression load $\bar{P}$ rendering the weak-form Equation (9) to admit a nontrivial solution for the deflection (i.e., $\bar{v} \neq 0$ ).

Now, the optimization problem becomes to find the maximum value of the normalized flexural buckling load $\bar{P}$ of the column subjected to the following normalized volumetric constraint:

$$
\int_{0}^{1} \bar{A}(\bar{x}) d \bar{x}=1
$$




\section{Discretization}

A standard finite element method is adopted together with the power method to determine the normalized buckling load and the corresponding buckling shape of the column for any profile of the normalized cross-sectional area $\bar{A}=\bar{A}(x)$. A gradient descent algorithm is implemented along with the representation of the normalized cross-sectional area $\bar{A}=\bar{A}(x)$ by piecewise polynomial functions to determine the optimal solution for the buckling load. Details of such implementations are briefly summarized below.

The column occupying the interval $[0,1]$ in the normalized space is first partitioned into $n$ finite elements such that $[0,1]=\underset{e=1, n}{\cup} \Omega_{e}$ where $\Omega_{e}=\left[\bar{x}_{e-1}, \bar{x}_{e}\right], \bar{x}_{0}=0$, and $\bar{x}_{n}=1$. The normalized deflection and the weight function over a generic element $\Omega_{e}$ are approximated by:

$$
\bar{v}^{e}\left(\bar{x}^{e}\right)=\boldsymbol{N}^{e} \boldsymbol{u}^{e} ; \quad \bar{w}^{e}\left(\bar{x}^{e}\right)=\boldsymbol{N}^{e} \boldsymbol{w}^{e}
$$

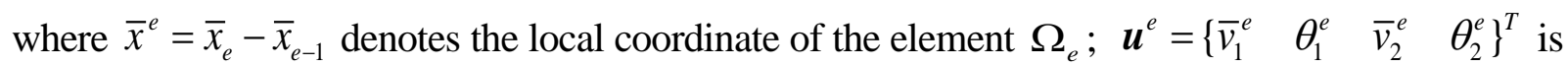
a vector containing the normalized end displacements $\bar{v}_{1}^{e}, \bar{v}_{2}^{e}$ and the end rotations $\theta_{1}^{e}, \theta_{2}^{e} ; \boldsymbol{w}^{e}$ is an arbitrary vector; and $N^{e}=N^{e}\left(\bar{x}^{e}\right)$ is a row matrix containing standard hermite shape functions:

$$
N^{e}=\left[1-3 \xi^{2}+2 \xi^{3} \quad \bar{h}^{e} \xi(1-\xi)^{2} \quad \xi^{2}(3-2 \xi) \quad \bar{h}^{e} \xi^{2}(\xi-1)\right]
$$

where $\bar{h}^{e}$ is the length of the element $\Omega_{e}$ and $\xi=\bar{x}^{e} / \bar{h}^{e}$. The normalized cross-sectional area $\bar{A}$ of the element $\Omega_{e}$ can also be approximated by:

$$
\bar{A}^{e}\left(\bar{x}^{e}\right)=\sum_{i=1}^{m} \varphi_{i}^{e}\left(\bar{x}^{e}\right) \bar{A}_{i}^{e}
$$

where $m$ denotes the number of interpolation points within the element $\Omega_{e} ; \bar{A}_{1}^{e}, \bar{A}_{2}^{e}, \ldots, \bar{A}_{m}^{e}$ are normalized cross-sectional area at interpolation points; and $\varphi_{1}^{e}, \varphi_{2}^{e}, \ldots, \varphi_{m}^{e}$ are linearly independent polynomial interpolation functions of degree $m-1$ defined over the element $\Omega_{e}$. For $m=1, \varphi_{1}^{e}$ is simply a constant function and the interpolation point is chosen at the midpoint of the element, whereas for $m=2, \varphi_{1}^{e}, \varphi_{2}^{e}$ are two independent linear functions and the interpolation points are chosen at both ends of the element. The interpolation functions for $m=1$ and $m=2$ are given by:

$$
\begin{array}{ll}
m=1: & \varphi_{1}^{e}\left(\bar{x}^{e}\right)=1 \\
m=2: & \varphi_{1}^{e}\left(\bar{x}^{e}\right)=1-\xi, \quad \varphi_{2}^{e}\left(\bar{x}^{e}\right)=\xi
\end{array}
$$


The approximated cross-sectional areas over the entire column for $m=1$ and $m=2$ are also shown in Figure 2. By substituting the approximation (11) and (13) over the finite element mesh $\underset{e=1, n}{\cup} \Omega_{e}$ into the weak-form Equation (9), it leads to a system of homogeneous, linear algebraic equations governing the approximate normalized buckling load $\bar{P}$ :

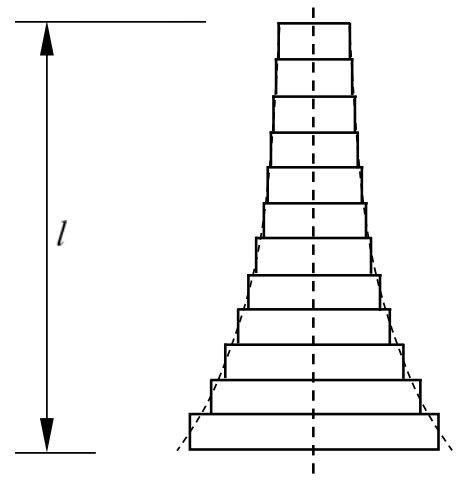

$m=1$

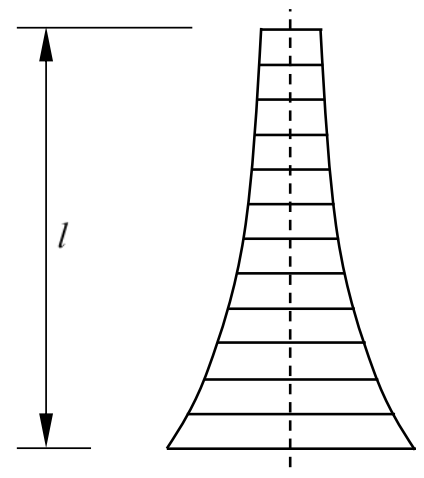

$m=2$

Figure 2. Discretization of normalized cross-sectional area using piecewise constant $(m=1)$ and piecewise linear $(m=2)$ interpolation functions

$$
(\boldsymbol{K}-\bar{P} \boldsymbol{M}) \boldsymbol{U}=\mathbf{0}
$$

where $\boldsymbol{U}$ denotes a vector containing all degrees of freedom of the discretized column at the onset of the buckling, and $\boldsymbol{K}, \boldsymbol{M}$ are matrices defined by:

$$
\begin{gathered}
\boldsymbol{K}=\sum_{e=1}^{n} \boldsymbol{k}^{e}, \quad \boldsymbol{k}^{e}=\hat{\boldsymbol{k}}^{e}+\sum_{i=1}^{m} \sum_{j=1}^{m} \overline{\boldsymbol{k}}_{i j}^{e} \bar{A}_{i}^{e} \bar{A}_{j}^{e}, \\
\hat{\boldsymbol{k}}^{e}=\int_{\Omega_{e}} \bar{k}^{e} \boldsymbol{N}^{e}\left(\boldsymbol{N}^{e}\right)^{T} d \bar{x}^{e}, \overline{\boldsymbol{k}}_{i j}^{e}=\int_{\Omega_{e}} \varphi_{i}^{e} \varphi_{j}^{e} \boldsymbol{C}^{e}\left(\boldsymbol{C}^{e}\right)^{T} d \bar{x}^{e} \\
\boldsymbol{M}=\sum_{e=1}^{n} \boldsymbol{m}^{e}, \quad \boldsymbol{m}^{e}=\int_{\Omega_{e}} \boldsymbol{B}^{e}\left(\boldsymbol{B}^{e}\right)^{T} d \bar{x}^{e}
\end{gathered}
$$

in which $\boldsymbol{B}^{e}=d \boldsymbol{N}^{e} / d \bar{x}^{e}, \boldsymbol{C}^{e}=d \boldsymbol{B}^{e} / d \bar{x}^{e}$, and the summations appearing in (16) and (17) imply the direct assembly of element contribution via the standard procedure. It is worth noting that the matrices $\boldsymbol{m}^{e}, \hat{\boldsymbol{k}}^{e}$, and $\overline{\boldsymbol{k}}_{i j}^{e}$ can be readily obtained in a closed form via the direct integration. Upon the representation (13), the volumetric constraint (10) becomes:

$$
\boldsymbol{F} \bar{A}=1
$$


where $\bar{A}$ is a vector storing the normalized cross-sectional areas at all interpolation points and $\boldsymbol{F}$ is a row matrix defined by:

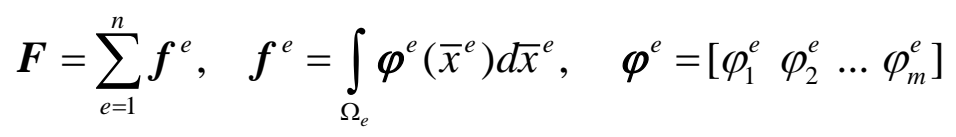

where, again, the summation appearing in (19) is carried out via the direct assembly procedure and the explicit expressions of $\boldsymbol{f}^{e}$ can also be obtained via the direct integration. Now, the statement of the discretized problem is to maximize the buckling load $\bar{P}$ satisfying Equation (15) and subjected to the volumetric constraint (18).

\section{Solution Scheme}

An iterative procedure based upon the standard power method together with Rayleigh quotient is adopted to determine the buckling load $\bar{P}$ of the eigen-system (15) for any prescribed data of the normalized cross-sectional area at all interpolation points $\bar{A}$. The least eigenvalue is estimated by forming Rayleigh quotient and the convergence indicator is then computed. The specified tolerance of $10^{-9}$ is employed in the present study for checking the convergence criterion. If the criterion is satisfied, the iteration process is terminated and the least eigenvalue is obtained.

To maximize the buckling load $\bar{P}$ subjected to the volumetric constraint (18), a projected gradient descent method is implemented. Specifically, by starting from an initial value of $\overline{\boldsymbol{A}}^{(0)}$, the projected gradient descent iterates the following condition until a convergence condition is satisfied:

$$
\overline{\boldsymbol{A}}^{(k+1)}=\mathcal{P}_{Q}\left(\overline{\boldsymbol{A}}^{(k)}+\gamma^{(k)} \nabla \overline{\boldsymbol{P}}\left(\overline{\boldsymbol{A}}^{(k)}\right)\right)
$$

where $Q$ is a set containing all $\bar{A}$ satisfying the constraint (18), $\gamma^{(k)}>0$ denotes the step size, $\nabla \bar{P}\left(\overline{\boldsymbol{A}}^{(k)}\right)$ is the gradient of $\bar{P}$ evaluated at $\overline{\boldsymbol{A}}^{(k)}$, and $\mathcal{P}_{Q}$ is the projection operator defined by

$$
\mathcal{P}_{Q}\left(\overline{\boldsymbol{A}}^{*}\right)=\arg \min _{\overline{\boldsymbol{A}} \in Q} \frac{1}{2} \sum_{i=1}^{N}\left(\bar{A}_{i}-\bar{A}_{i}^{*}\right)^{2}
$$

with $N$ denoting the number of interpolation points. For the constraint (18), it can be shown that

$$
\mathcal{P}_{Q}\left(\overline{\boldsymbol{A}}^{*}\right)=\overline{\boldsymbol{A}}^{*}+\left(\frac{1-\boldsymbol{F} \overline{\boldsymbol{A}}^{*}}{\boldsymbol{F} \boldsymbol{F}^{T}}\right) \boldsymbol{F}^{T}
$$

Another nontrivial task is to determine the gradient of the normalized buckling load with respect to the normalized cross-sectional area at all interpolation points. Before proceeding, it is remarked that the eigenvector $U$ corresponding to the buckling load $\bar{P}$ in Equation (15) is unique only up to the scaling magnitude (i.e., if $\boldsymbol{U}$ is an eigenvector, then $\alpha \boldsymbol{U}$ is also the 
eigenvector for any $\alpha \neq 0$ ). As a result, one of its entries can be set, without loss, to a unity. By taking a partial derivative of Equation (15) with respect to the normalized cross-sectional area at each interpolation point, it leads to:

$$
(\boldsymbol{K}-\bar{P} \boldsymbol{M}) \frac{\partial \boldsymbol{U}}{\partial \bar{A}_{i}}-\frac{\partial \bar{P}}{\partial \bar{A}_{i}} \boldsymbol{M U}=-\frac{\partial \boldsymbol{K}}{\partial \bar{A}_{i}} \boldsymbol{U}
$$

where $\bar{A}_{i}$ denote the normalized cross-sectional area at the $i^{\text {th }}$ interpolation point contained in the vector $\bar{A}$. By multiplying equation (23) by $\boldsymbol{U}^{T}$ and then enforcing the condition (15), it leads to:

$$
\frac{\partial \overline{\boldsymbol{P}}}{\partial \bar{A}_{i}}=\frac{\boldsymbol{U}^{T}\left(\partial \boldsymbol{K} / \partial \bar{A}_{i}\right) \boldsymbol{U}}{\boldsymbol{U}^{T} \boldsymbol{M U}}
$$

Equation (24) is sufficient for determining the gradients $\partial \bar{P} / \partial \bar{A}_{i}$ once the normalized buckling load $\bar{P}$ and the corresponding eigenvector $U$ are obtained from the power method. The gradient $\partial \boldsymbol{K} / \partial \bar{A}_{i}$ can be obtained, in a closed form, as:

$$
\begin{gathered}
\frac{\partial \boldsymbol{K}}{\partial \bar{A}_{i}}=\sum_{e=1}^{n} \frac{\partial \boldsymbol{k}^{e}}{\partial \bar{A}_{i}} \\
\frac{\partial \boldsymbol{k}^{e}}{\partial \bar{A}_{i}}= \begin{cases}\frac{\partial \boldsymbol{k}^{e}}{\partial \bar{A}_{p_{i}}^{e}}=2 \sum_{q=1}^{m} \bar{A}_{q}^{e} \overline{\boldsymbol{k}}_{q p_{i}}^{e} & \text { if the } i^{\text {th }} \text { interpolation point } \in \Omega_{e} \\
\mathbf{0} & \text { if the } i^{\text {th }} \text { interpolation point } \notin \Omega_{e}\end{cases}
\end{gathered}
$$

where $p_{i}$ denote the local numbering of the $i^{\text {th }}$ interpolation point in the element $\Omega_{e}$. It is evident from (25) and (26) that the computation of $\partial \boldsymbol{K} / \partial \bar{A}_{i}$ involves only elements containing the $i^{\text {th }}$ interpolation point.

\section{Numerical Results}

Results obtained from the proposed technique are first compared with those reported in the literature to confirm the validity of the formulation and implementations. A selected set of results for more complex cases is then presented to further demonstrate its capability and the convergence behavior of numerical solutions. In the numerical study, uniform meshes with either piecewise constant $(m=1)$ or piecewise linear $(m=2)$ interpolation functions for the representation of the cross-sectional area are employed.

\section{Case 1: C-F and H-H Columns}

Consider, first, the column with the clamped-free $(\mathrm{C}-\mathrm{F})$ and hinged-hinged $(\mathrm{H}-\mathrm{H})$ end conditions as shown in Figure 3. The exact solutions for the optimal cross-sectional area and the maximum buckling load of these two columns were reported in the work of [20] and used, here, as the benchmark solutions. 
The computed maximum buckling loads normalized by the exact solution are reported in Table 1 for both $\mathrm{C}-\mathrm{F}$ and $\mathrm{H}-\mathrm{H}$ columns and $m=1$ and 2, and the corresponding profiles of the normalized cross-sectional area are, also, shown in Figure 4 and 5. It is seen that the convergence of numerical solutions is confirmed as the mesh is refined, and the converged maximum buckling load and the corresponding normalized cross-sectional area agree very well with the benchmark solution for both types of the column. This, therefore, confirm the validity of the implemented scheme and the good convergence behavior of the numerical solutions.

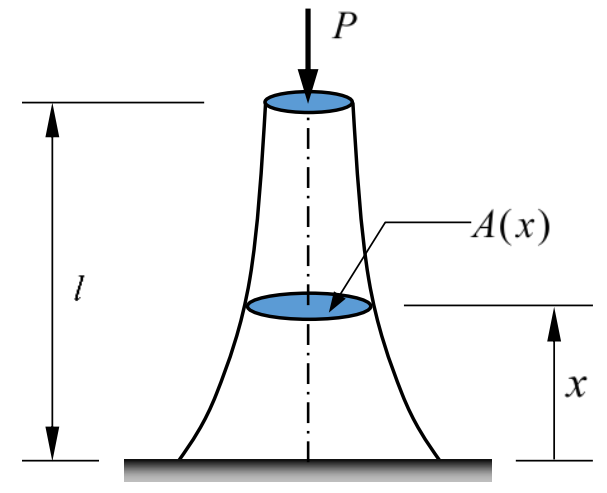

C-F Column

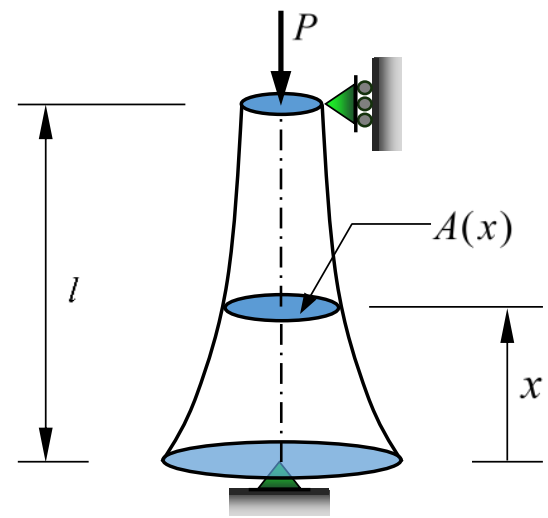

H-H Column

Figure 3. Schematic of perfectly straight, clamped-free (C-F) and hinged-hinged (H-H) columns

In addition, for the same level of accuracy, using the piecewise linear interpolation function $(m=2)$ to represent the variation of the normalized cross-sectional area requires significantly less number of elements than that for the piecewise constant interpolation function $(m=1)$. In particular, for $m=2$, only 2 elements (4 elements) are sufficient to generate the optimal solution for C-F column (H-H column) with the error less than $1 \%$, whereas for $m=1$, the mesh containing at least 16 elements ( 32 elements) is required to generate the solution of the same level of accuracy.

Finally, we remark by passing that a problem of the $\mathrm{H}-\mathrm{H}$ column of the length $l$ can be reduced, via the symmetry, to an equivalent problem of the $\mathrm{C}-\mathrm{F}$ column of the length $l / 2$; as a result, to generate the optimal solution for the H-H column with the same accuracy as that for the C-F column of the same length, it requires twice the number of elements as clearly indicated in Table 1.

\section{Case 2: H-H Column with a Concentrated Elastic Spring Installed at Mid Height}

Consider, again, the hinged-hinged $(\mathrm{H}-\mathrm{H})$ column with a concentrated, translational, elastic spring with a constant $k_{0}$ being stalled at the mid height as illustrated in Figure 6. The influence of the elastic spring on the optimal profile of the cross-sectional area and the maximum buckling load is investigated.

Within the context of the current formulation, this concentrated spring can be readily handled by taking $k(x)=k_{0} \delta(x-l / 2)$ with $\delta(x-l / 2)$ denoting the Dirac-delta function centered at $x=l / 2$. 
Table 1. Normalized Maximum Buckling Load for C-F and H-H Column Obtained from Different Meshes and $m=1$ and 2. Exact Maximum Buckling Loads for Both Cases are Obtained from [20].

\begin{tabular}{|c|c|c|c|c|}
\hline \hline \multirow{2}{*}{$n$} & \multicolumn{4}{|c|}{$\bar{P}_{\text {current }} / \bar{P}_{\text {exact }}$} \\
\cline { 2 - 5 } & \multicolumn{2}{|c|}{ C-F Column } & \multicolumn{2}{c|}{ H-H Column } \\
\cline { 2 - 5 } & $m=1$ & $m=2$ & $m=1$ & $m=2$ \\
\hline 2 & 0.868682 & 0.991737 & 0.755642 & 0.973455 \\
\hline 4 & 0.941946 & 0.997588 & 0.868682 & 0.991737 \\
\hline 8 & 0.977041 & 0.999288 & 0.941946 & 0.997588 \\
\hline 16 & 0.991515 & 0.999786 & 0.977041 & 0.999288 \\
\hline 32 & 0.996997 & 0.999935 & 0.991515 & 0.999786 \\
\hline 64 & 0.998968 & 0.999980 & 0.996997 & 0.999935 \\
\hline 128 & 0.999653 & 0.999994 & 0.998968 & 0.999980 \\
\hline \hline
\end{tabular}

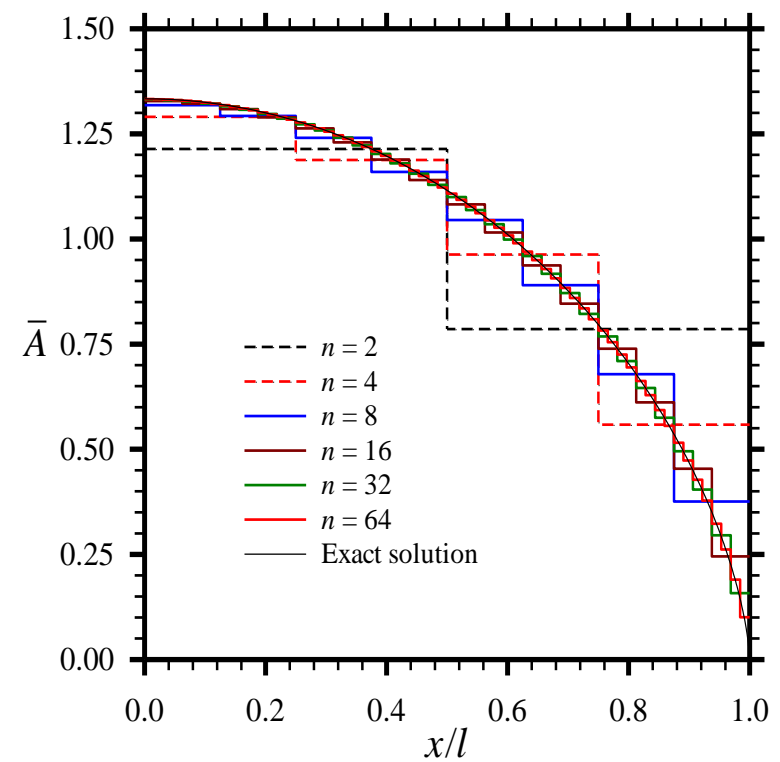

(a)

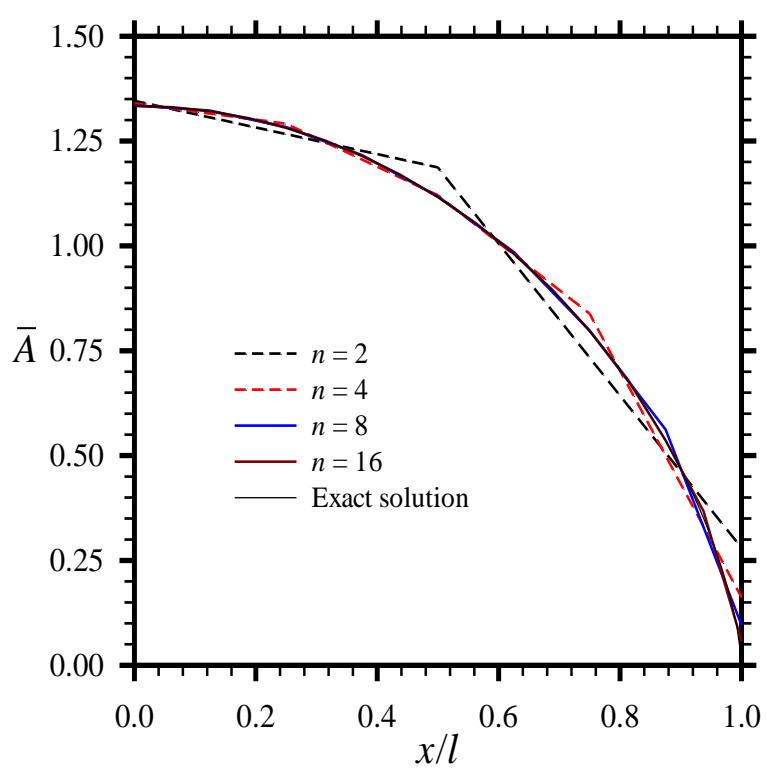

(b)

Figure 4. Profile of normalized cross-sectional area at the maximum normalized buckling load for C-F column: (a) $m=1$ and (b) $m=2$. The profiles for $n=128$ for $m=1$ and $n=32$,

64, 128 for $m=2$ are intentionally excluded from the two plots since they are nearly indistinguishable from the exact solutions 


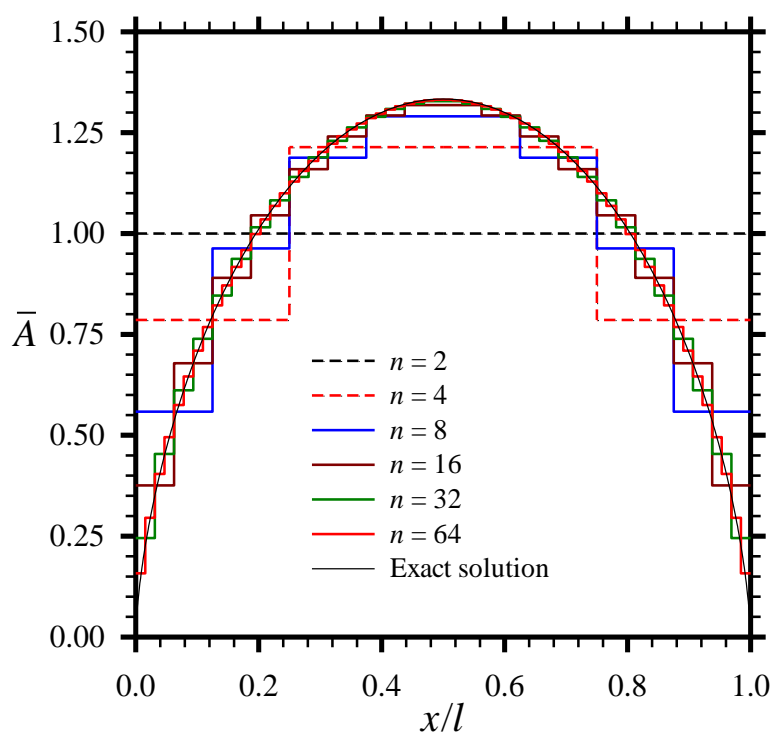

(a)

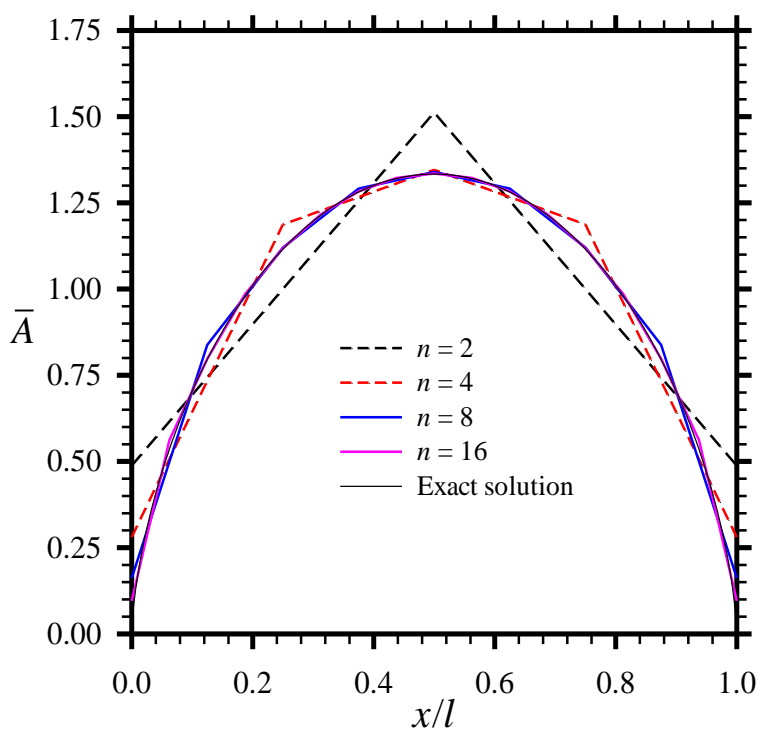

(b)

Figure 5. Profile of normalized cross-sectional area at the maximum normalized buckling load for H-H column: (a) $m=1$ and (b) $m=2$. The profiles for $n=128$ for $m=1$ and $n=32,64,128$ for $m=2$ are intentionally excluded from the two plots since they are nearly indistinguishable from the exact solutions
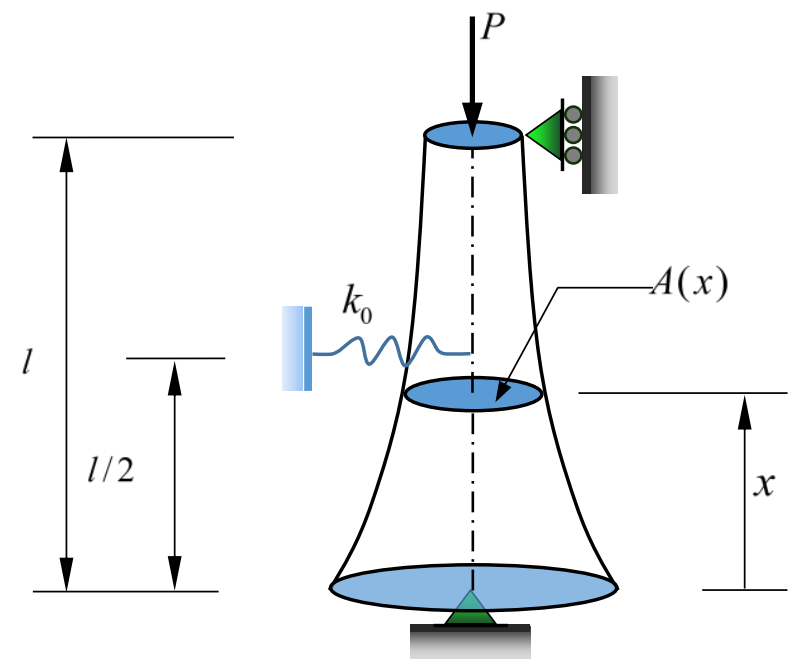

\section{H-H Column}

Figure 6. Schematic of a perfectly straight, hinged-hinged $(\mathrm{H}-\mathrm{H})$ column with a concentrated elastic spring installed at mid height

In the numerical study, the normalized cross-sectional area is represented by both the piecewise constant and linear interpolation functions (i.e., $m=1,2$ ) and a series of uniform meshes is adopted to ensure the convergence of the numerical solutions. Table 2 shows the computed maximum buckling load for various values of the normalized spring constant $\bar{k}_{0}=4 \pi k_{0} l^{5} / E V_{0}^{2}$ 
and the number of elements used in the discretization. It is seen that the converged results can be achieved via the mesh refinement and, again, the number of elements required to obtain the converged optimal solution is less for the case $m=2$.

The ratio between the maximum normalized buckling load $(\bar{P})$ and that for the $\mathrm{H}$ H column without the elastic spring $\left(\bar{P}_{H H}\right)$, and the profile of the optimal normalized crosssectional area $\bar{A}$ obtained from $n=128$ and $m=2$ are reported in Figure 7(a) and 7(b), respectively, as a function of the normalized spring constant $\bar{k}_{0}$. It is evident that $\bar{P}$ increases almost linearly with respect to $\bar{k}_{0}$. It is also evident from results in Figure 7 (b) that the profile of the optimal normalized cross-sectional area is strongly dependent on the value of $\bar{k}_{0}$ especially in the region close to the location where the elastic spring is installed. In particular, the profile of the normalized cross-sectional area is non-smooth at that location and the value of $\bar{A}$ decays as $\bar{k}_{0}$ increases.

Table 2. Normalized Maximum Buckling Load for H-H Column with Elastic Spring Installed at Mid Height. Results are Obtained for Various Meshes and Normalized by the Maximum Normalized Buckling Load for H-H Column Without Elastic Spring (i.e., $\left.\bar{k}_{0}=0\right)$.

\begin{tabular}{|c|c|c|c|c|c|c|}
\hline \hline \multirow{2}{*}{$n$} & \multicolumn{2}{|c|}{$\bar{P}_{\text {current }} / \bar{P}_{H H}$} \\
\cline { 2 - 7 } & \multicolumn{2}{|c|}{$\bar{k}_{0}=10$} & \multicolumn{2}{|c|}{$\bar{k}_{0}=50$} & \multicolumn{2}{c|}{$\bar{k}_{0}=150$} \\
\cline { 2 - 7 } & $m=1$ & $m=2$ & $m=1$ & $m=2$ & $m=1$ & $m=2$ \\
\hline 2 & 0.911891 & 1.109072 & 1.532142 & 1.656319 & 3.039636 & 3.051745 \\
\hline 4 & 1.008114 & 1.128467 & 1.563130 & 1.680325 & 2.906125 & 3.094231 \\
\hline 16 & 1.079061 & 1.134601 & 1.630428 & 1.687474 & 3.020562 & 3.104633 \\
\hline 32 & 1.128505 & 1.136899 & 1.681176 & 1.690130 & 3.096570 & 3.108184 \\
\hline 64 & 1.134059 & 1.137053 & 1.687077 & 1.690305 & 3.104347 & 3.108396 \\
\hline 128 & 1.136065 & 1.137100 & 1.689234 & 1.690358 & 3.107079 & 3.108459 \\
\hline \hline
\end{tabular}




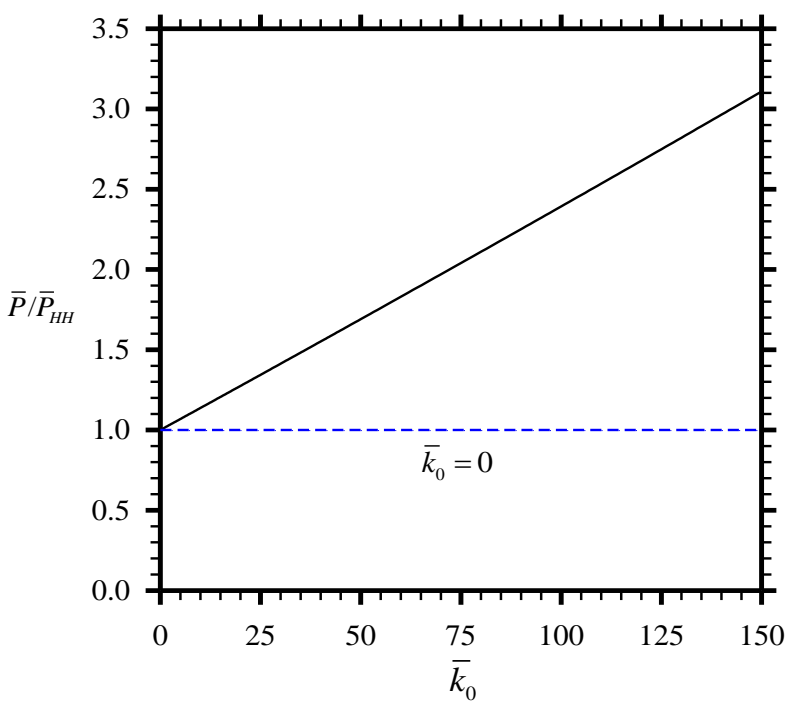

(a)

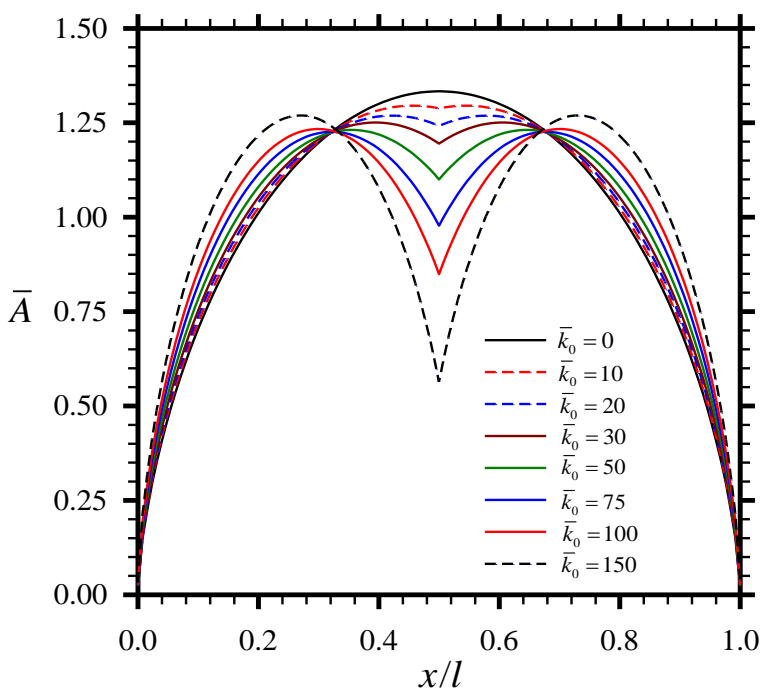

(b)

Figure 7. (a) Normalized maximum buckling load versus the normalized spring constant and (b) profile of the optimal normalized cross-sectional area of hinged-hinged $(\mathrm{H}-\mathrm{H})$ column with the elastic spring installed at the mid height

\section{Case 3: C-F Column with a Uniformly Distributed Elastic Spring}

Finally, consider a clamped-free (C-F) column restrained against the transverse displacement by means of a distributed elastic spring with the spring constant $k(x)=k_{0}$ where $k_{0}$ is a given constant as shown schematically in Figure 8 . The key interest, here, is to investigate the influence of the spring constant $k_{0}$ on the maximum buckling load and the profile of the optimal cross-sectional area of the column. In the convergence study, a series of uniform meshes is adopted and, again, both the piecewise constant and piecewise linear interpolation functions $(m$ $=1,2$ ) are employed to represent the normalized cross-sectional area of the column. Computed maximum buckling loads normalized by that of the same column without the elastic spring ( $\left.k_{0}=0\right)$ are reported in Table 3 for $\bar{k}_{0} \equiv 4 \pi k_{0} l^{6} / E V_{0}^{2} \in\{5,10,25\}$ and $n \in\{2,4,8,16,32,64,128\}$

. Similar to the previous cases, the converged solutions can be achieved for all values of the normalized spring constant treated as the mesh is uniformly refined, and only few elements are required to obtain such accurate results if the piecewise linear interpolation function $(m=2)$ is utilized to discretize the cross-sectional area.

The maximum normalized buckling load $(\bar{P})$ obtained from $n=128$ and $m=2$ is normalized by that of the clamped-free (C-F) column without the lateral restraint $\left(\bar{P}_{C F}\right)$ and then reported as a function of the normalized spring constant $\left(\bar{k}_{0}\right)$ in Figure 9(a). It is seen that the maximum normalized buckling load increases almost proportionally to the normalized spring constant similar to the previous case. In addition, the uniform lateral restraint significantly influences the variation of the normalized cross-sectional area at the optimality condition as can be observed in Figure 9(b). In particular, the cross-sectional area at the clamped end decays as $\bar{k}_{0}$ increases. The increase in the normalized spring constant tends to shift the location of maximum normalized cross-sectional area to an interior point far away from the clamped end. 


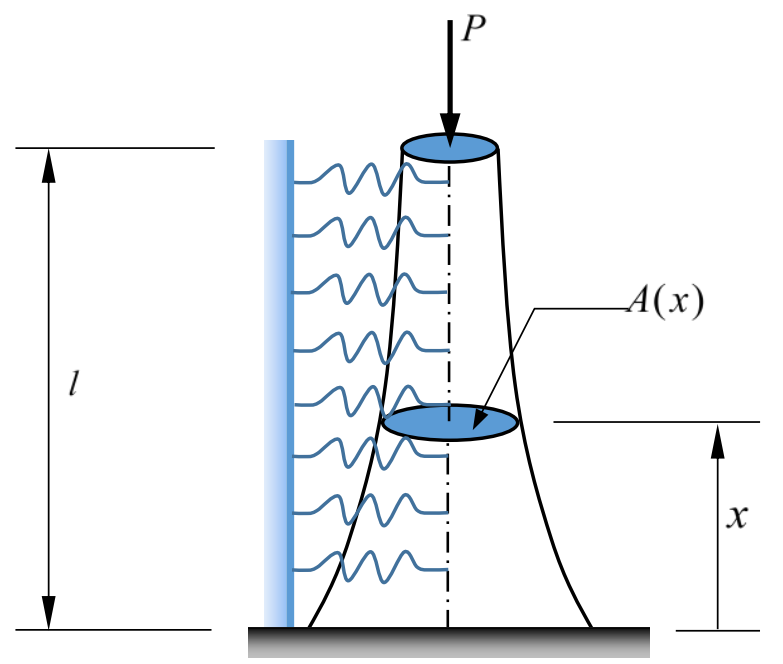

Figure 8. Schematic of a perfectly straight, clamped-free (C-F) column restrained against the transverse displacement by means of a distributed elastic spring of constant $k_{0}$ over its entire length.

Table 3. Normalized Maximum Buckling Load for Clamped-Free (C-F) Column Transversely Restrained by Distributed Elastic Spring over its Entire Length. Results are Obtained for $m=2$ and Various Meshes, and Normalized by the Maximum Normalized Buckling Load for Clamped-Free (C-F) Column Without Elastic Spring.

\begin{tabular}{|c|c|c|c|c|c|c|}
\hline \multirow{2}{*}{$n$} & \multicolumn{6}{|c|}{$\bar{P}_{\text {current }} / \bar{P}_{C F}$} \\
\cline { 2 - 7 } & \multicolumn{2}{|c|}{$\bar{k}_{0}=5$} & \multicolumn{2}{|c|}{$\bar{k}_{0}=10$} & \multicolumn{2}{c|}{$\bar{k}_{0}=25$} \\
\cline { 2 - 7 } & $m=1$ & $m=2$ & $m=1$ & $m=2$ & $m=1$ & $m=2$ \\
\hline 2 & 1.087626 & 1.199671 & 1.304183 & 1.413430 & 1.921837 & 2.094392 \\
\hline 4 & 1.151585 & 1.206640 & 1.364892 & 1.421428 & 2.019963 & 2.106278 \\
\hline 8 & 1.185730 & 1.208657 & 1.399672 & 1.423731 & 2.074971 & 2.109513 \\
\hline 36 & 1.200501 & 1.209237 & 1.415097 & 1.424384 & 2.097746 & 2.110357 \\
\hline 64 & 1.206254 & 1.209407 & 1.421193 & 1.424574 & 2.106184 & 2.110590 \\
\hline 128 & 1.209101 & 1.209474 & 1.424244 & 1.424648 & 2.110175 & 2.110678 \\
\hline \hline
\end{tabular}




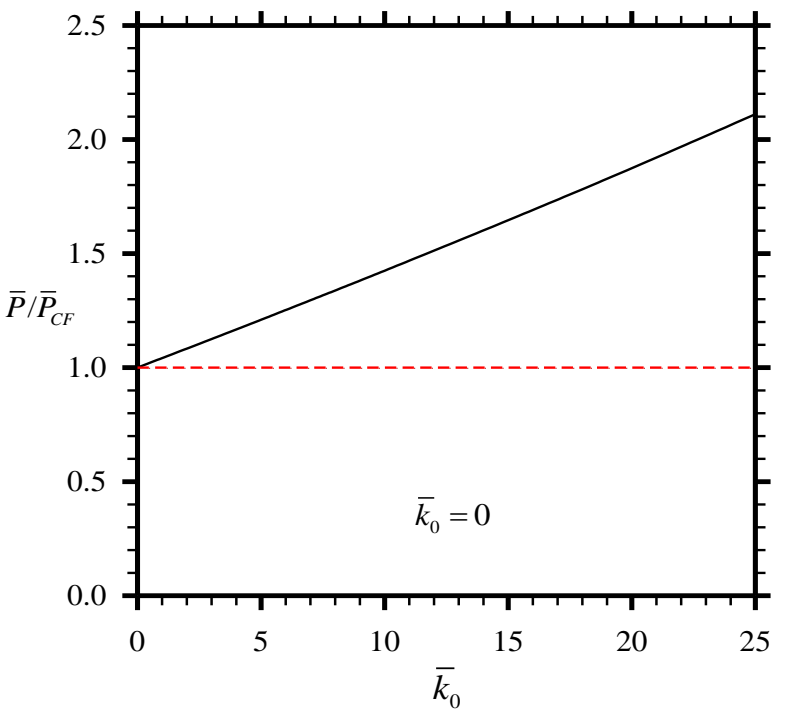

(a)

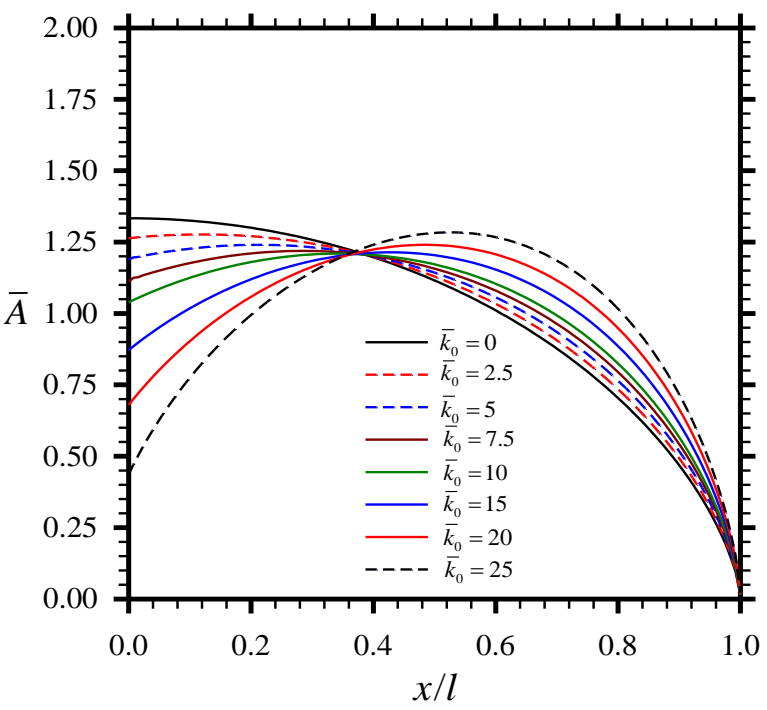

(b)

Figure 9. (a) Normalized maximum buckling load versus the normalized spring constant and (b) profile of optimal normalized cross-sectional area of C-F column transversely restrained by distributed elastic spring over its entire length

\section{Conclusions}

A simple technique based upon the standard finite element approximation and projected gradient descent scheme has been successfully implemented for determining the optimal profile of the cross-sectional area to achieve the maximum elastic flexural buckling load of a perfectly straight column with/without the lateral restraints subjected to the constraint on the material volume. The profile of the cross-sectional area has been represented by piecewise polynomial interpolation functions defined locally on the same mesh used in the discretization of the buckled shape. The least eigenvalue and the corresponding eigenvector of a discretized linear system have been efficiently obtained from the power method together with Rayleigh quotient. The information of the gradient and projection operator required in the projected gradient descent algorithm has been obtained in an explicit fashion.

Results from an extensive numerical study have confirmed the convergence, accuracy, and capability of the proposed technique. In particular, using the linear shape functions to represent the variation of the cross-sectional area over each element yield the better convergence of the optimal solutions than using the constant shape functions. While the technique has been developed and proved computationally promising within a quite limited context of the single modal formulation and columns with only the volumetric constraint, the concept and essential components can be further extended to handle bimodal/multimodal formulation, more complicated and large-scale structures, and various constraint conditions.

\section{Acknowledgement}

This research is supported by The Thailand Research Fund (Grant No. RTA6280012). The first author also gratefully acknowledges the supports provided by the Faculty of Engineering and Architecture, Rajamangala University of Technology Tawan-ok, Bangkok, Thailand. 


\section{References}

[1] M.J. Kochenderfer, and T.A. Wheeler, Algorithms for Optimization, The MIT Press, Massachusetts, United States, 2019.

[2] O. Hasançebi, "Optimization of truss bridges within a specified design domain using evolution strategies," Engineering Optimization, Vol. 39, No. 6, pp. 737-756, 2007. doi: https://10.1080/03052150701335071

[3] V.K. Koumousis, and P.G. Georgiou, "Genetic algorithms in discrete optimization of steel truss roofs," Journal of Computing in Civil Engineering, Vol. 8, No. 3, pp. 309325, 1994. doi: https://10.1061/(ASCE)0887-3801(1994)8:3(309)

[4] C.S. Krishnamoorthy, P.P. Venkatesh, and R. Sudarshan, "Object-oriented framework for genetic algorithms with application to space truss optimization," Journal of Computing in Civil Engineering, Vol. 16, No. 1, pp. 66-75, 2002. doi: https://10.1061/(ASCE)0887-3801(2002)16:1(66)

[5] L.J. Li, Z.B. Huang, F. Liu, and Q.H. Wu, "A heuristic particle swarm optimizer for optimization of pin connected structures," Computers \& Structures, Vol. 85, No. 7, pp. 340-349, 2007. doi: https://doi.org/10.1016/j.compstruc.2006.11.020

[6] S. Rajeev, and C.S. Krishnamoorthy, "Discrete optimization of structures using genetic algorithms," Journal of Structural Engineering, Vol. 118, No. 5, pp. 1233-1250, 1992. doi: https://10.1061/(ASCE)0733-9445(1992)118:5(1233)

[7] J.F. Schutte, and A.A. Groenwold, "Sizing design of truss structures using particle swarms," Structural and Multidisciplinary Optimization, Vol. 25, No. 4, pp. 261-269, 2003. doi: https://10.1007/s00158-003-0316-5

[8] V. Toğan, and A.T. Daloğlu, "An improved genetic algorithm with initial population strategy and self-adaptive member grouping," Computers \& Structures, Vol. 86, No. 11, pp. 1204-1218, 2008. doi: https://doi.org/10.1016/j.compstruc.2007.11.006

[9] E. Doğan, and M.P. Saka, "Optimum design of unbraced steel frames to LRFD-AISC using particle swarm optimization," Advances in Engineering Software, Vol. 46, No. 1, pp. 27-34, 2012. doi: https://doi.org/10.1016/j.advengsoft.2011.05.008

[10] W. Li, Q. Li, G.P. Steven, and Y.M. Xie, “An evolutionary approach to elastic contact optimization of frame structures," Finite Elements in Analysis and Design, Vol. 40, No. 1, pp. 61-81, 2003. doi: https://doi.org/10.1016/S0168-874X(02)00179-8

[11] W. Liu, and J. Ye, "Collapse optimization for domes under earthquake using a genetic simulated annealing algorithm," Journal of Constructional Steel Research, Vol. 97, pp. 59-68, 2014. doi: https://doi.org/10.1016/j.jcsr.2014.01.015

[12] C. Perea, J. Alcala, V. Yepes, F. Gonzalez-Vidosa, and A. Hospitaler, "Design of reinforced concrete bridge frames by heuristic optimization," Advances in Engineering Software, Vol. 39, No. 8, pp. 676-688, 2008. doi: https://doi.org/10.1016/j.advengsoft.2007.07.007

[13] S. Pezeshk, C.V. Camp, and D. Chen, "Design of nonlinear framed structures using genetic optimization," Journal of Structural Engineering, Vol. 126, No. 3, pp. 382-388, 2000. doi: https://10.1061/(ASCE)0733-9445(2000)126:3(382)

[14] S. Talatahari, A.H. Gandomi, X.-S. Yang, and S. Deb, "Optimum design of frame structures using the Eagle Strategy with Differential Evolution," Engineering Structures, Vol. 91, pp. 16-25, 2015. doi: https://doi.org/10.1016/j.engstruct.2015.02.026 
[15] I. Kusano, A. Baldomir, J. Ángel Jurado, and S. Hernández, "Probabilistic optimization of the main cable and bridge deck of long-span suspension bridges under flutter constraint," Journal of Wind Engineering and Industrial Aerodynamics, Vol. 146, pp. 59-70, 2015. doi: https://doi.org/10.1016/j.jweia.2015.08.001

[16] I. Kusano, M. Cid Montoya, A. Baldomir, F. Nieto, J.Á. Jurado, and S. Hernández, "Reliability based design optimization for bridge girder shape and plate thicknesses of long-span suspension bridges considering aeroelastic constraint," Journal of Wind Engineering and Industrial Aerodynamics, Vol. 202, p. 104176, 2020. doi: https://doi.org/10.1016/j.jweia.2020.104176

[17] F. J. Martínez, F. González-Vidosa, A. Hospitaler, and V. Yepes, "Heuristic optimization of RC bridge piers with rectangular hollow sections," Computers \& Structures, Vol. 88, No. 5, pp. 375-386, 2010. doi: https://doi.org/10.1016/j.compstruc.2009.11.009

[18] L. Sgambi, K. Gkoumas, and F. Bontempi, "Genetic algorithms for the dependability assurance in the design of a long-span suspension bridge," Computer-Aided Civil and Infrastructure Engineering, Vol. 27, No. 9, pp. 655-675, 2012. doi: https://10.1111/j.1467-8667.2012.00780.x

[19] J.B. Keller, "The shape of the strongest column," Archive for Rational Mechanics and Analysis, Vol. 5, pp. 275-285, 1960.

[20] I. Tadjbakhsh, and J.B. Keller, "Strongest columns and isoperimetric inequalities for eigenvalues," Journal of Applied Mechanics, Vol. 29, No. 1, pp. 159-164, 1962. doi: https://doi.org/10.1115/1.3636448

[21] G.J. Simitses, M.P. Kamat, and C.V. Smith, "Strongest column by the finite element displacement method," AIAA Journal, Vol. 11, No. 9, pp. 1231-1232, 1973. doi: https://10.2514/3.50571

[22] N. Olhoff, and S.H. Rasmussen, "On single and bimodal optimum buckling loads of clamped columns," International Journal of Solids and Structures, Vol. 13, No. 7, pp. 605-614, 1977. doi: https://doi.org/10.1016/0020-7683(77)90043-9

[23] Y. Tada, and L. Wang, "Reinvestigation on optimization of clamped-clamped columns and symmetry of corresponding eigenfunctions," JSME International Journal Series A Solid Mechanics and Material Engineering, Vol. 38, No. 1, pp. 38-43, 1995. doi: https://10.1299/jsmea1993.38.1_38

[24] D. Manickarajah, Y.M. Xie, and G.P. Steven, "Optimisation of columns and frames against buckling," Computers \& Structures, Vol. 75, No. 1, pp. 45-54, 2000. doi: https://doi.org/10.1016/S0045-7949(99)00082-6

[25] S.G. Krishna, and Y.M. Ram, "Discrete model analysis of optimal columns," International Journal of Solids and Structures, Vol. 44, No. 22, pp. 7307-7322, 2007. doi: https://doi.org/10.1016/j.ijsolstr.2007.04.010

[26] H. Zhang, C.M. Wang, N. Challamel, and E. Ruocco, "Semi-analytical solutions for optimal design of columns based on Hencky bar-chain model," Engineering Structures, Vol. 136, pp. 87-99, 2017. doi: https://doi.org/10.1016/j.engstruct.2017.01.011

[27] E. Ruocco, C.M. Wang, H. Zhang, and N. Challamel, "An approximate model for optimizing Bernoulli columns against buckling," Engineering Structures, Vol. 141, pp. 316-327, 2017. doi: https://doi.org/10.1016/j.engstruct.2017.01.077 